\title{
PALEOFAUNA DE AGUACALIENTE DE CARTAGO, COSTA RICA PARTE I: EQUUS CF. E. CONVERSIDENS OWEN, 1869
}

\author{
Ana Lucía Valerio Z. ${ }^{1}$ \\ César Alberto Laurito M. ${ }^{2}$ \\ ${ }^{1}$ Departamento de Historia Natural, Museo Nacional de Costa Rica \\ Apartado Postal 749-1000, San José - Costa Rica \\ E-mail: alvalerio@costarricense.cr \\ ${ }^{2}$ Instituto Nacional de Aprendizaje, Núcleo de Turismo, \\ Apartado postal 203-2200, Coronado; San José, Costa Rica \\ E-mail: cesarlaurito@ice.co.cr
}

(Recibido 25/5/05; Aceptado 11/7/05)

\begin{abstract}
An isolated tooth of Equus cf. E. conversidens Owen is recorded for the first time from the Upper Pleistocene of Costa Rica and Central America. This premolar was found with some molars of an unidentified ruminant. This species is very common in the Pleistocene of North America and was firstly described for the Valley of Mexico. The Costa Rican found represents the most austral distribution of the species.

Keywords: Perissodactyla, Equidae, Equus, Biotic Interchange, Costa Rica.

RESUMEN: Por primera vez se registra la especie Equus cf. E. conversidens para el Pleistoceno de Costa Rica y de América Central. El hallazgo se fundamenta en un único premolar superior y se encontró asociado a varios molares de un rumiante no determinado. Esta especie es muy común en el Pleistoceno de Norte América, y fue inicialmente descrita para el valle de México. El hallazgo costarricense representa la distribución más austral de la especie.

Palabras clave: Perissodactyla, Equidae, Equus, Pleistoceno Superior, Intercambio Biótico, Costa Rica.
\end{abstract}

\section{INTRODUCCIÓN}

La presencia de vertebrados fósiles en la zona de Aguacaliente se remonta al año 1921, cuando se estaban realizando las obras de la planta eléctrica de Cartago. En ese entonces se localizaron en el río Aguacaliente restos fósiles de un vertebrado en el suelo aluvial a $1,5 \mathrm{~m}$ de profundidad (Tristán, 1921). El mismo autor indica la presencia de costillas, vérte- bras, porciones de extremidades, una parte del cráneo, molares y una gran variedad de fragmentos indeterminados, que sugieren pertenecían a un mastodonte.

En el año de 1999, en las cercanías de Aguacaliente, fueron recuperadas varias piezas dentales por el Hno. Alfonso Lázaro Alcalde de la Orden Agustinos Recoletos de la Ciudad de los Niños, cuando se realizaban las obras de construcción de la Urbanización Hacienda de 
Oro. Entre los restos dentales se conserva un premolar $\mathrm{P}^{2}$ de caballo bien preservado, objeto de estudio en el presente trabajo y varios restos de un rumiante aún en estudio.

El sitio de hallazgo se ubica en las siguientes coordenadas 202.400 y 545.200 Lambert, hoja cartográfica Istarú (IGNCR 3445 IV), escala 1:50,000 (Fig. 1).
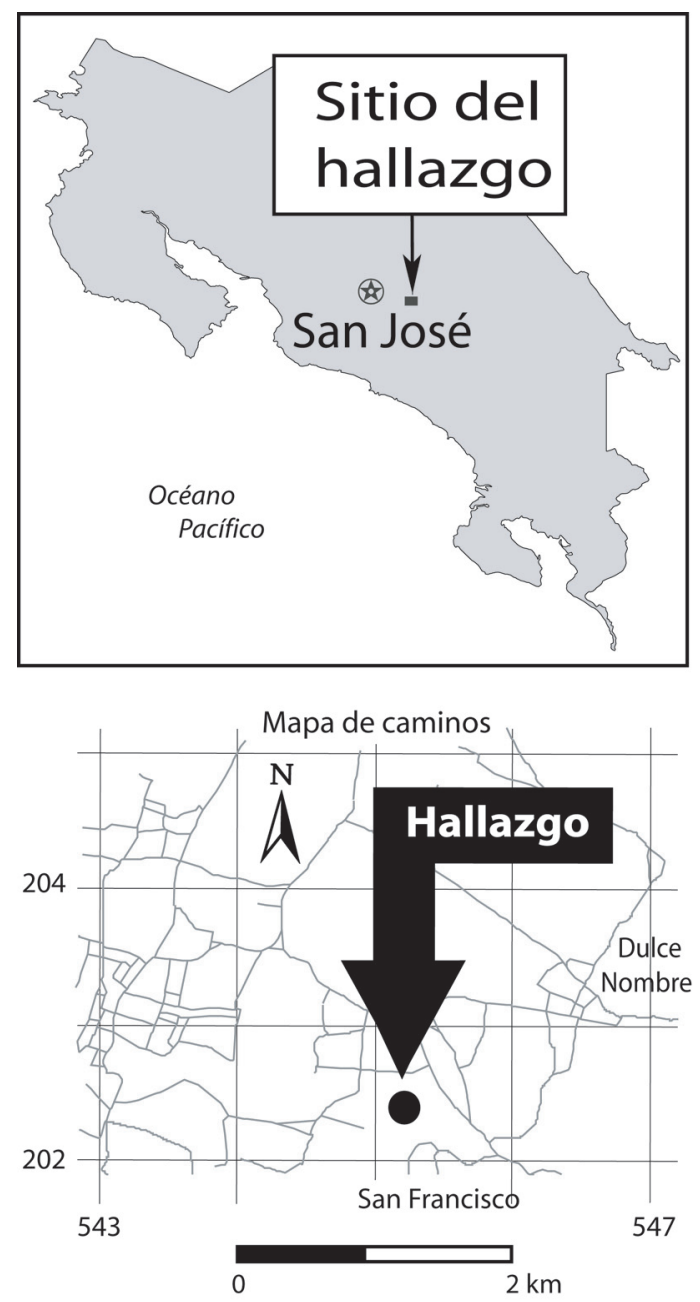

Fig. 1: Mapa de ubicación del sitio de hallazgo, Aguacaliente de Cartago.

\section{ESTRATIGRAFÍA}

Badilla et al. (1999) diferencian los depósitos no consolidados del Cuaternario en coluvios, lahares y aluviones. Los coluvios se localizan en las márgenes de los ríos Navarro y Aguacaliente y corresponden con un depósito brechoso muy mal seleccionado, con clastos angulares a subredondeados de composición variada. Estos se encuentran incluidos en una matriz areno arcillosa de coloración variada entre anaranjado, café y rojo, con restos de materia orgánica. Tanto los clastos como la matriz muestran una intensa meteorización. Los lahares se distribuyen en los alrededores de Tejar y San Francisco y presentan bloques métricos de andesitas basálticas, provenientes del volcán Irazú. Los aluviones se distribuyen a lo largo de los ríos Navarro, Aguacaliente, Patarrá y Sombrero; formando varios niveles de terrazas y están constituidos por bloques de arenitas cuarzosas, arenitas volcaniclásticas, lavas e intrusivos.

\section{PALEONTOLOGÍA}

Equus cf. E. conversidens Owen, 1869

Sinonimia:

Equus conversidens Owen, 1869 - Gidley, J.W., 1901: pág. 118, lám. XXI.

Asinus conversidens (Owen), 1869 - Quinn, J.H., 1957: págs 27-28, lám. III, figs. 3-9.

Asinus conversidens (Owen), 1869 -Mooser, O., 1959: págs. 443-444, fig. 24.

Equus (Hemionus) conversidens Owen, 1869 Skinner, 1972, pág. 125.

Equus conversidens Owen, 1869 - Kurtén, B. \& Anderson, E., 1980: pág. 291, figs. 14.1-3.

Equus conversidens Owen, 1869 - MontellanoBallesteros, M., 1990: págs. 200-201.

Equus conversidens Owen, 1869 - Czaplewzki, N.J., 1999:pág. 17-18.

Equus conversidens Owen, 1869 - Alberdi et al., 2003: pág. 211.

Equus conversidens Owen, 1869 - Azzaroli, 1998: pág. 8-9; lám. 14, figs. 1 a-c.

Reynoso-Rosales \& Montellano-Ballesteros (1994), toman como sinónimas 9 de las 10 especies descritas inicialmente por Mooser (1959) y sugieren que la población de équidos del Pleistoceno de Aguascalientes, México, pueden 
ser referidas a la especie Equus conversidens Owen, 1869, fundamentándose en análisis cuantitativos y cualitativos multivariados. Sin embargo, aún deben revisarse los datos, pues estos mismos autores sugieren la posibilidad de la existencia probable de al menos dos especies.

Posteriormente, Azzaroli (1998), sinonimisa la especie Onager zoyatalis Mosser, 1959 de la Fauna Cedazo; pero esta especie fue previamente incluida por Winans (1989) dentro del Grupo Equus francisi.

Material: un molar $\mathrm{P}^{2}$, bien preservado, depositado en la colección de fósiles del Museo Nacional, bajo el código CFM-1425.
Descripción: premolar superior con superficie

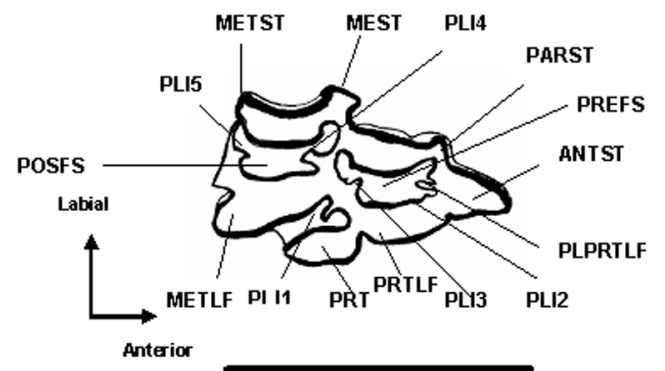

$3,5 \mathrm{~cm}$

Fig. 2: patrón oclusal del P2 de Equus conversidens Owen, 1869; ver descripción en el texto, simbología adaptada de Gidley, 1901 y diversos autores.

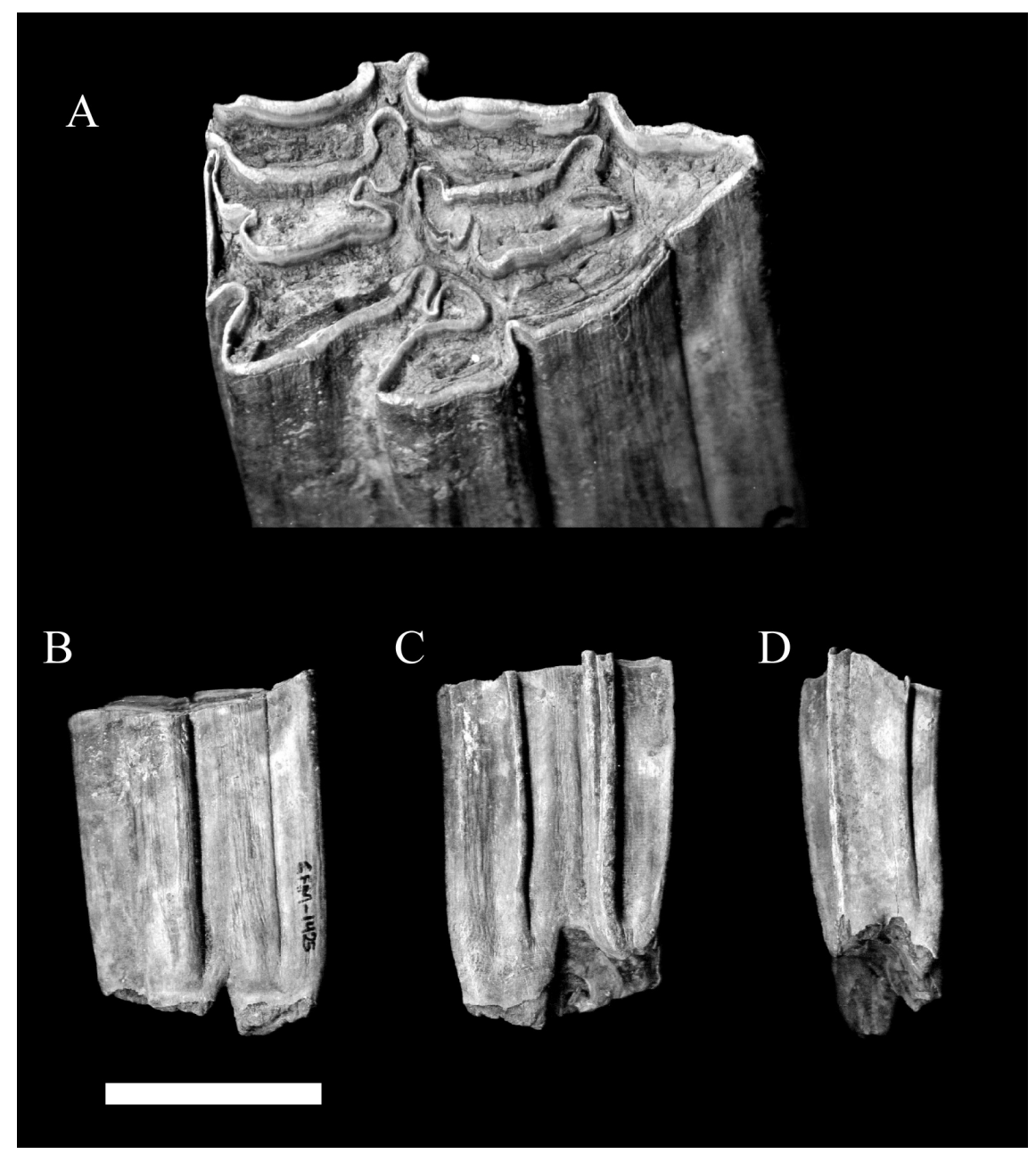

Fig. 3: P2 de Equus cf. E. conversidens Owen, 1869; en vista: a. oclusal, b. lingual, c. vestibular y d. comisural (escala visual = $3 \mathrm{~cm})$. 
oclusal de contorno triangular (Figs. 2 y 3), su longitud anteroposterior $35,0 \mathrm{~mm}$ y ancho vestíbulolingual 22,0 $\mathrm{mm}$, protocono PRT medianamente elongado y unido al protolofo PRTLF, lo que confirma su condición Equinae y con una longitud de $9 \mathrm{~mm}$. Posfoseta POSFS y prefosesta PREFS angostas en sentido transversal y ligeramente alargadas en sentido mesiodistal, con lobulaciones y pliegues simples; anterostilo ANTST triangular simple con el extremo redondeado; metastilo METST y mesostilo MEST angulares y parastilo PARST agudo. Metalofo MRTLF amplio y redondeado.

Pli-caballín PLI1 corto pero conspicuo; Plilingual de la prefoseta PLI2, regular muy poco desarrollado; Pli-prefoseta PLI3 (= pli-protoconulo), constituido por una lobulación simple al igual que con los Pli-posfoseta, PLI4 y Plihipostilo PLI5. Pli-protolofo, PLPRTLF, constituido por un pliegue corto y muy angosto.

\section{COMENTARIOS}

La especie Equus conversidens Owen, 1869, está basada en un paladar procedente del Pleistoceno Tardío del Valle de Tequixquiac, Estado de México.

Para muchos autores como Miller \& Carranza-Castañeda (1987), MontellanoBallesteros (1990), Reynoso-Rosales \& Montellano-Ballesteros (1994) y Azzaroli (1998), aún es válida la existencia de Equus conversidens Owen, 1869.

Por otra parte, solamente la subespecie Equus conversidens leoni, Stock, 1953 de la cueva de San Josecito, Nuevo León, México fue incluida dentro del grupo de Equus alaskae Hay, 1913 por Winans (1989), quien considera a $E$. alaskae como una de las pocas especies válidas para el Irvingtoniano y Rancholabreano (= Pleistoceno) de América del Norte; ello lo fundamenta esta última autora en un análisis multivariado de caracteres cuantitativos del cráneo, mandíbula y metapodiales (ver también ArroyoCabrales \& Johnson, 2003).

Winans (1989) al momento de analizar todos los materiales fósiles de Equus sp., incluyendo los de La Fauna Cedazo, Aguascalientes, México, tomó para su muestra los especimenes cuyos cráneos, ramos mandibulares y metapodiales estuviesen completos. Sin embargo, como aclaran Alberdi et al., 2003, la taxonomía que aporta Winans (1989) es aproximativa y se precisa de una revisión de las formas norteamericanas.

Teniendo en cuenta lo anterior, decidimos mantener la taxonomía tradicional e incluimos dentro de la especie Equus conversidens Owen el material de Aguacaliente de Cartago; lo anterior porque las dimensiones y el patrón dental del premolar en estudio es similar al de los ejemplares descritos para el Valle de México.

\section{Distribución paleogeográfica}

La especie E. conversidens Owen, se ha descrito para el Pleistoceno de la Fauna Cedazo, Aguascalientes, (Mooser, 1958; ReynosoRosales \& Montellano-Ballesteros, 1994) y la Localidad de El Cedral, San Luis Potosí (Alberdi et al., 2003) y la fauna local de "El Golfo" del Pleistoceno Medio del Noroeste del Estado de Sonora (Shaw, 1981 en Lindsay, 1984) todas de la República Mexicana. Pleistoceno de Arizona, Nuevo México, Midland, Canyon y Slaton, Texas (Quinn, 1957) y para el Pleistoceno Temprano de Nebraska y de la Formación Seymour, Knox, Texas (Skinner, 1972 y Dalquest \& Carpenter, 1988). También, en el Pleistoceno Tardío de Papago Springs Cave, Arizona (Czaplewski et al.,1999). La especie fue descrita inicialmente para el Valle de México y el hallazgo de Aguacaliente de Cartago en Costa Rica constituye el registro más austral y el primero de América Central.

\section{Edad}

Azzaroli (1998), señala que la especie $E$. conversidens Owen, se restringe al Pleistoceno Tardío del Sur de los Estados Unidos y a la República Mexicana.

La edad del hallazgo de Aguacaliente de Cartago, se considera como Rancholabreana, ya 
que en esa misma localidad y asociada a los suelos aluviales locales, constituidos por arcilla gris con fragmentos andesíticos, se recuperó una hemimandíbula parcial de mastodonte junto con otros restos craneales y postcraneales asociados. Estos últimos, fueron descritos inicialmente por Tristán (1921), los que luego Laurito (1988) determinó como pertenecientes a Cuvieronius hyodon Fischer (1814).

Asociado al premolar de E. cf. conversidens Owen, se encontraron varios molares y premolares, que se corresponden con al menos dos individuos de un rumiante que aún no ha sido determinado, pero que presentan el mismo tipo de conservación que el del premolar aquí descrito.

\section{CONCLUSIONES}

Se describe el hallazgo de un premolar superior de E. cf. conversidens Owen, procedente de la localidad de Aguacaliente de Cartago, Costa Rica. Este hallazgo representa la distribución más austral de la especie en mención.

La edad del mismo se considera Pleistoceno Superior Tardío, correspondiente con el NALMA es Rancholabreano, fundamentado principalmente en su asociación con Cuvieronius hyodon Fischer (1814) y un rumiante aún no determinado, aunque la distribución de la especie es Pleistoceno en general.

\section{AGRADECIMIENTOS}

Al Dr. Joaquín Arroyo Cabrales del Instituto Nacional de Antropología e Historia de México, quien gentilmente aportó material bibliográfico y quien junto a la Dra. María Teresa Alberdi del Departamento de Paleobiología del Museo Nacional de Ciencias Naturales de España, aportaron valiosos comentarios, nuestro más sincero agradecimiento. De igual forma al Profesor Dr. Walter Landini del Dipartamento Di Scienze Della Terra, Universidad de Pisa, por el importante aporte de bibliografía a ésta investigación.

\section{REFERENCIAS}

ALBERDI, M.T., ARROYO-CABRALES, J. \& POLACO, O.J., 2003: ¿Cuántas especies de caballo hubo en una sola localidad del Pleistoceno Mexicano. - Rev. Española Paleont. 18(2): 205-212.

ARROYO-CABRALES, J. \& JONSON, E., 2003: Catálogo de los ejemplares procedentes de la cueva de San Josecito, Nuevo León, México. Univ. Nal. Autón. México, Inst. Geol. Revista 20(1): 79-93.

AZZAROLI, A., 1998: The genus Equus in North America - The Pleistocene species. Paleontographica Italica, 85: 1-60.

BADILLA, E., LINKIMER, L. \& ZÚÑIGA, H., 1999: Geología de Navarro y alrededores, provincia de Cartago, Costa Rica. - 100 págs. Univ. de Costa Rica, 100 págs. [Inf. Campaña Geol.].

CZAPLEWSKI, N.J., MEAD; J.I., BELL, C.J., PEACHEY, W.D. \& KU, T., 1999: Papago Spring cave revisited, Part II: Vertebrate paleofauna. - Occasional Pap. Oklahoma Mus. Nat. Hist. 5: 1-41.

DALQUEST, W.W. \& HUGHES, J.T., 1965: The Pleistocene horse, Equus conversidens. - The Amer. Midland Naturalist, 74: 408-417.

DALQUEST, W.W. \& CARPENTER, R.M., 1988: Early Pleistocene (Irvingtonian) mammals from the Seymour Formation, Knox and Baylor Counties, Texas, exclusive of Camelidae. - 28 págs. Occasional Pap. Museum Texas Tech. Univ. 124.

GIDLEY, J.W., 1901: Tooth characters and revision of the North American species of the genus Equus. - Bull. Amer. Mus. Nat. Hist. 14: $91-142$.

KURTÉN, B. \& ANDERSON, E., 1980: Pleistocene mammals of North America. - 442 págs. Columbia Univ. Press. 
LAURITO, C., 1988: Los proboscidios fósiles de Costa Rica y su contexto en América Central. Vínculos, 14(1-2): 29-58.

LINDSAY, E.H., 1984: Late Cenozoic mammals from Northwestern Mexico. - En: MacFadden, B.J. (ed.): Origin and evolution of the Cenozoic vertebrate fauna of Middle America. - J. Vertebrate Paleont. 4(2): 208-215.

MILLER, W. \& CARRANZA-CASTAÑEDA, O., 1984: Late Cenozoic mammals from Central México. - J. Vertéebrate Paleont. 4:

MONTELLANO-BALLESTEROS, M., 1990: Una edad del Irvingtoniano al Rancholabreano para la fauna Cedazo del Estado de Aguascalientes. Univ. Nal. Autón. México, Inst. Geol. Rev. 9(2): 195-203.

MOOSER, O., 1959: La fauna "Cedazo" del Pleistoceno de Aguascalientes. - Anales del Inst. Biología 29(1-2): 409-452, [1958].

QUINN, J.H., 1957: Pleistocene Equidae of Texas. 51 págs. Bureau of Econ. Geol. Report of Investigations, 33 .
REYNOSO-ROSALES, V.H. \& MONTELLANOBALLESTEROS, M., 1994: Revisión de los équidos de la fauna Cedazo del Pleistoceno de Aguascalientes, México. - Univ. Nal. Autón. México, Inst. Geol. Rev. 11(1): 87-105.

SKINNER, M.F., 1972: Order Perissodactyla. - En: SKINNER, M.F. \& HIBBARD, C.W. (eds.): Early Pleistocene pre-glacial and glacial rocks and faunas of north-central Nebraska. - Bull. Amer. Mus. Nat. Hist. 148: 1-148.

STOCK, C., 1953: El Caballo Pleistoceno (Equus conversidens leoni subsp. nov.) de la cueva de San Josecito, Aramberri, Nuevo León. - Mem. Congr. Cient. Mexicano, Univ. Nal. Autón. México T III: 170.

TRISTÁN, J.F., 1921: Notas sobre los restos de un vertebrado fósil hallado en Agua Caliente de Cartago. - Rev. de Costa Rica, 2: 337-340.

WINANS, M.C., 1989: A quantitative study of North American fossil species of the genus Equus. En: PROTHERO, D.R. \& SCHOCH, R.M. (eds.): The evolution of perissodactyls. Clarendon Press-Oxford Univ. Press, 262-297. 